OPEN ACCESS

Edited by:

Hua Feng,

Army Medical University, China

Reviewed by:

Jason H. Huang,

Baylor Scott and White Health,

United States

Günalp Uzun,

Gülhane Askerî Tip Akademisi, Turkey

*Correspondence:

Jing Yang

yangjinghbo@ccmu.edu.cn

Nan Kang

cyyygk2020@126.com

Specialty section:

This article was submitted to

Neurotrauma

a section of the journal

Frontiers in Neurology

Received: 18 May 2020

Accepted: 31 August 2020

Published: 15 October 2020

Citation:

Liu X, Liang F, Zhang J, Li Z, Yang J and Kang N (2020) Hyperbaric Oxygen Treatment Improves Intestinal

Barrier Function After Spinal Cord Injury in Rats.

Front. Neurol. 11:563281.

doi: 10.3389/fneur.2020.563281

\section{Hyperbaric Oxygen Treatment Improves Intestinal Barrier Function After Spinal Cord Injury in Rats}

\author{
Xuehua Liu ${ }^{1}$, Fang Liang ${ }^{1}$, Jing Zhang ${ }^{1}$, Zhuo Li ${ }^{1}$, Jing Yang ${ }^{1 *}$ and Nan Kang ${ }^{2 *}$ \\ 1 Department of Hyperbaric Oxygen Medicine, Beijing Chaoyang Hospital, Capital Medical University, Beijing, China, \\ ${ }^{2}$ Department of Orthopedic Surgery, Beijing Chaoyang Hospital, Capital Medical University, Beijing, China
}

Intestinal barrier dysfunction is often observed clinically after spinal cord injury (SCI) and seriously affects long-term quality of life. Hyperbaric oxygen (HBO) treatment has been proved to promote barrier function recovery after injury, but the influence of $\mathrm{HBO}$ on intestinal barrier function following $\mathrm{SCl}$ is unclear. We aimed to investigate the effect and mechanisms of $\mathrm{HBO}$ treatment on intestinal barrier function by measuring the level of tight junction (TJ) proteins and the Ras homolog (Rho)/Rho-associated coiled-coil forming protein kinase (ROCK) signaling pathway. SCl model was established in rats, and the animals were randomly assigned into three groups: sham-operation group $(\mathrm{SH})$, $\mathrm{SCl}$ group and $\mathrm{SCl}+\mathrm{HBO}$ group. In the $\mathrm{SCl}+\mathrm{HBO}$ group, the rats inhaled $100 \% \mathrm{O}_{2}$ for $1 \mathrm{~h}$ at 2.0 atmospheres absolute pressure (ATA) once per day after surgery. Neurological function and intestinal permeability were assessed after surgery, and the jejunum tissue was excised for histological and intestinal barrier function evaluations. The protein levels of TJ and the Rho/ROCK signaling pathway were also measured. The results showed that in the SCl group, intestinal mucosal injury score, intestinal permeability, and levels of Rho and ROCK1 were higher, and TJ proteins occludin and ZO-1 were lower than those in the $\mathrm{SH}$ group $(P<0.01)$. HBO treatment significantly inhibited the expression of Rho and ROCK1, increased occludin and ZO-1 expression, decreased intestinal permeability, and alleviated intestinal mucosal injury as compared with the SCI group $(P<0.05, P<0.01)$. The $\mathrm{SCl}+\mathrm{HBO}$ group showed higher Basso-Beattie-Bresnahan (BBB) scores relative to the $\mathrm{SCl}$ group on postoperative days 7 and $14(P<0.01)$. There was a significant negative correlation between BBB score and intestinal mucosal injury score in rats after $\mathrm{HBO}$ treatment $(P<0.05)$. We concluded from this study that $\mathrm{HBO}$ treatment promoted the expression of $\mathrm{TJ}$ proteins possibly through inhibiting Rho/ROCK signaling pathway, which protected the intestinal barrier function and improved the intestinal permeability after $\mathrm{SCl}$ in rats.

Keywords: hyperbaric oxygen, intestinal barrier, spinal cord injury, tight junction protein, Rho/ROCK signaling pathway 


\section{INTRODUCTION}

Apart from the disastrous sensory and motor losses following spinal cord injury (SCI), dysfunction of a variety of organ systems is also widely recognized, such as the gastrointestinal (GI) tract, lung, kidney, spleen, and liver $(1,2)$. GI complications, e.g., barrier dysfunction, motility disorders, and microbial dysbiosis, reprehensively account for $11 \%$ of hospitalizations in SCI patients and are regarded as serious problems compromising quality of life $(3,4)$. Intestinal barrier dysfunction facilitates translocation of luminal bacteria into the circulation and mesenteric lymph, leading to life-threatening systemic inflammatory response syndrome (SIRS) and multiple organ dysfunction syndrome (MODS) (5). So far, some studies have reported intervention strategies to improve intestinal barrier function following SCI, such as melatonin, sacral nerve electrostimulation, and probiotics, which could promote intestinal peristalsis and suppress bacterial translocation (6-8). Hence, protection of intestinal barrier function is vital for intestinal homeostasis and the prognosis of SCI individuals.

Hyperbaric oxygen (HBO) refers to the inhalation of $100 \%$ pure oxygen in an environment $>1$ absolute atmospheric (ATM) pressure. Multiple studies reported that $\mathrm{HBO}$ treatment promoted barrier recovery after various injuries. $\mathrm{HBO}$ treatment could enhance the arterial partial pressure of oxygen $\left(\mathrm{PaO}_{2}\right)$, increase the oxygen content, improve the perfusion of ischemia tissue, and stabilize the blood-brain barrier after cerebral ischemia/reperfusion injury $(9,10)$. Yang et al. demonstrated that HBO treatment stabilized the blood-spinal cord barrier by downregulating the expression of MMP-2 and MMP-9 following SCI (11). However, the influence of HBO treatment on intestinal barrier function following SCI is unclear, and the underlying mechanism needs to be clarified.

Tight junction (TJ) proteins, including claudin, occludin, and ZO-1, are the important components of intestinal barrier, which regulate the passage of ions and solutes, maintain cell polarity, and are the key determinants of the paracellular permeability (12-14). Ras homolog (Rho) belongs to G protein family, which circulates between an inactive guanosine diphosphate (GDP)-bound and an active guanosine triphosphate (GTP)bound state and then regulates cellular activity through acting on its downstream target molecular, Rho-associated coiled-coil forming protein kinase (ROCK) (15). The Rho/ROCK signaling pathway has been proved to be an important regulator of TJ protein function $(16,17)$.

In present study, we aimed to investigate the effect and mechanisms of HBO treatment on intestinal barrier function after SCI in rats. We hypothesized that $\mathrm{HBO}$ treatment may promote the expression of $\mathrm{TJ}$ proteins through regulating the Rho/ROCK signaling pathway and may then protect intestinal barrier function.

\section{MATERIALS AND METHODS}

\section{Animals}

All surgical operation and postoperative care were performed according to the ethical principles laid down by the Committee for Experimental Animal Welfare and Ethics, Capital Medical University. Healthy male Sprague-Dawley rats $(n=144$, purchased from the Experimental Animals Center of Capital Medical University, Beijing, China) $\geq 8$ weeks of age, weighing 250-300 g, were used in the experiment. The rats were feed with enough food and water in a stable temperature $\left(23^{\circ} \mathrm{C}\right)$ and humidity (60\%) with a 12:12-h light-dark cycle. Each rat was randomly assigned into three experimental groups: sham-operation group (SH), SCI group, or SCI+HBO group. Meanwhile, the experimental animals were also randomly assigned into four postoperative times (1, 3, 7, and 14 days). This study did not involve pathogenic microorganisms and was carried out in a general secondary laboratory, and all animals are specific pathogen free.

\section{Spinal Cord Injury Model}

Contusive SCI was performed using a MASCIS (Multicenter Animal Spinal Cord Injury Study) impactor as described previously (18). The experimental rats were anesthetized using $10 \%$ chloral hydrate intraperitoneally $(350 \mathrm{mg} / \mathrm{kg})$ and underwent a laminectomy at level T10. The spinal cord dorsal was fully exposed and suffered a fall injury with a 10-g rod dropped from a height of $25 \mathrm{~mm}$. After injury, the wound was sutured layer by layer, and the rats were placed in single cage and accepted intensive care to recover from anesthetic and surgical operations. We assisted the rats to empty their bladder twice a day until the spontaneous urination reflex was established.

\section{Hyperbaric Oxygen Treatment}

Rats in the SCI+HBO group began to receive $\mathrm{HBO}$ treatment in an experiment hyperbaric chamber $6 \mathrm{~h}$ after operation. The chamber was flushed with $100 \% \mathrm{O}_{2}$ for $5 \mathrm{~min}$ to avoid carbon dioxide accumulation. Compression air was conducted to 2.0 atmospheres absolute pressure (ATA) for $10 \mathrm{~min}$ and maintained for $60 \mathrm{~min}$ at 2.0 ATA inhaling $100 \%$ oxygen, then decompression air was performed to normobaric air for $5 \mathrm{~min}$. According to the above treatment plan, rats received $\mathrm{HBO}$ treatment once a day. Rats in the SH and SCI groups inhaled $21 \%$ oxygen at 1.0 ATA postoperatively.

\section{Analysis of Locomotor Function}

Locomotor function of rats was evaluated by the Basso-BeattieBresnahan (BBB) score for locomotion on postoperative days 1, 3, 7 , and 14 . The BBB scale includes the movement of hindlimbs and joints, weight-bearing capability, limb coordination and gait, paw position, and tail height. The highest score is 21 . The behavior of rats was evaluated by two persons blinded to the experiment in an open field $(120 \times 120 \mathrm{~cm})$ for $5 \mathrm{~min}$.

\section{Assessment of Intestinal Permeability}

On postoperative day 3 , the intestinal permeability was evaluated by fluorescein isothiocyanate (FITC)-labeled dextran in the light of a method described previously (19). Briefly, rats $(n=12$ per group) were prohibited from eating and drinking for $4 \mathrm{~h}$ and were given $0.6 \mathrm{mg} / \mathrm{g}$ of FITC-dextran $(4 \mathrm{kDa}$; SigmaAldrich) dissolved in sterile phosphate-buffered saline (PBS) via gastric gavage prior to sacrifice. Four hours after gavage, 
the rats were anesthetized, and blood was collected via cardiac puncture. Specimens were centrifuged for $15 \mathrm{~min}$ at $3,000 \times g$. The plasma was then measured using spectrofluorophotometer at an excitation wavelength of $490 \mathrm{~nm}$ and an emission wavelength of $525 \mathrm{~nm}$.

\section{Tissue Harvest}

At designated times after injury, experimental rats were deeply anesthetized using chloral hydrate. The abdominal cavity was exposed through a midline incision, and the small intestine was carefully separated. A 2-cm length of middle jejunum was obtained and fixed with $4 \%$ paraformaldehyde solution for histopathological assessment. Another part of the jejunum was placed in liquid nitrogen for protein analysis after flushing with saline.

\section{Histological Assessment}

The jejunum was fixed in $4 \%$ paraformaldehyde overnight, flushed in PBS, and continuously dehydrated using low to high concentration of ethanol. After the ethanol in the tissue was replaced with xylene, tissue can be embedded in paraffin. After sectioning, 5- $\mu \mathrm{m}$ tissue sections were rehydrated again using orderly high to low concentration of ethanol; finally, the sections were put into the distilled water and stained with hematoxylin and eosin. Histological evaluation was performed by two pathologists blinded to this experiment protocol. The degree of intestinal mucosal injury was graded using a scale of $0-5$ as described previously (20): $0=$ intact intestinal villi and epithelium; 1 = subepithelial Gruenhagen's gap at the tip of villus, slight capillary dilation and congestion; $2=$ moderate dilation of intestinal subepithelial space, separation of mucosa, and lamina propria; $3=$ moderate to severe separation of mucosa and lamina propria, partial shedding of intestinal villi tips; $4=$ shedding of intestinal villi and lamina propria, exposed dilated capillaries; and $5=$ loss of intestinal villi, hemorrhage, and necrosis of lamina propria.

\section{Immunohistochemistry}

The 5 - $\mu \mathrm{m}$ tissue slices mentioned above were deparaffinized routinely; the slices were immersed in EDTA antigen repair buffer $(\mathrm{pH} 8.0)$ and heated in a microwave oven for antigen repair. After cooling naturally, the sections were washed with PBS ( $\mathrm{pH}$ 7.4). In order to block the endogenous peroxidase activity, the sections were incubated in the dark for $25 \mathrm{~min}$ at room temperature in $3 \%$ hydrogen peroxide and then were washed with $\mathrm{PBS}(\mathrm{pH}$ 7.4) again. The sections were incubated for overnight at $4^{\circ} \mathrm{C}$ with rabbit polyclonal anti-occludin (1:1,000, ab216327), rabbit polyclonal anti-ZO-1 (1:1,000, ab96587), rabbit monoclonal antiRho (1:1,000, ab40673), and rabbit monoclonal anti-ROCK1 (1:2,000, ab45171) (all from Abcam, Cambridge, MA, USA) as primary antibodies, then were added into horseradish peroxidase (HRP)-conjugated goat anti-rabbit IgG (Santa Cruz Biotechnology, Inc. USA) to cover tissue, and incubated for $50 \mathrm{~min}$ at room temperature. The sections were washed with PBS and added into DAB chromogenic liquid for color rendering, and the sections were counterstained with hematoxylin. The presence of brownish yellow granules was considered as positive protein expression. The average optical density (AOD) of positive staining in five fields per section and five sections per animal was analyzed using Image-Pro Plus 6.0 (Media Cybernetics, USA) software. All samples were analyzed by two double-blinded pathologists under high-times optical microscope.

\section{Western Blotting}

In order to prepare the protein sample, the frozen jejunum tissue was lysed using tissue protein lysis buffer, and the protein concentration was determined according to the instructions of the bicinchoninic acid (BCA) protein quantitative kit (Sun Bio, Beijing, China). Sodium dodecyl sulfate-polyacrylamide gels $(10-12 \%)$ were configured according to the weight of detected proteins. Fifty micrograms of protein in each sample was extracted to perform electrophoresis, transferred to nitrocellulose filter membranes, then was incubated overnight at $4^{\circ} \mathrm{C}$ with primary antibodies against occludin (1:1,000, ab216327), ZO1 (1:1,000, ab96587), Rho (1:1,000, ab40673), ROCK1 (1:2,000, ab45171), and $\beta$-actin (1:2,000) (all from Abcam, Cambridge, MA, USA). After being washed three times, the membranes were incubated with HRP-conjugated secondary antibodies (Santa Cruz Biotechnology, Inc. USA) for $2 \mathrm{~h}$ at $37^{\circ} \mathrm{C}$. Then the membranes were reacted using enhanced chemiluminescence (ECL) solution (Millipore, Corporation, Billerica, MA, USA), and then were scanned (Konica Minolta Medical Imaging, Inc., Wayne, NJ, USA). The expression of protein was quantitatively analyzed using target protein $/ \beta$-actin with Adobe Photoshop (Adobe, Mountain View, CA, USA) and Lab Works (UVP, Upland, CA, USA) software.

\section{Statistical Analysis}

The statistical analysis was performed by SPSS 19.0 (IBM Inc., Chicago, IL, USA) software. All data were expressed as the mean \pm standard deviation (SD). One-way analysis of variance (ANOVA) was applied to analyze the differences among experimental groups in intestinal mucosal injury score, FITC level, locomotor score, and the expression of TJ and Rho/ROCK signal proteins. Pearson's correlation coefficient was calculated to analyze the relationship between locomotor function and intestinal mucosal injury. $P<0.05$ was thought to be of significant difference in statistics.

\section{RESULTS}

\section{Hyperbaric Oxygen Treatment Maintains Intestinal Barrier Function After Spinal Cord Injury in Rats}

To evaluate the potential role of $\mathrm{HBO}$ treatment on intestinal mucosal histomorphology after SCI, the jejunum was removed from SCI rats at the appropriate times. As shown in Figure 1, compared with the $\mathrm{SH}$ group (Figure 1A), the jejunum mucosal tissue was compromised on day 3 following SCI (Figure 1B). For example, the villus height decreased significantly and the villi were disorganized, crypt depth increased significantly, the subepithelial interstice of villi was obvious, and upper cortex and solid layers were significantly detached. However, HBO treatment 

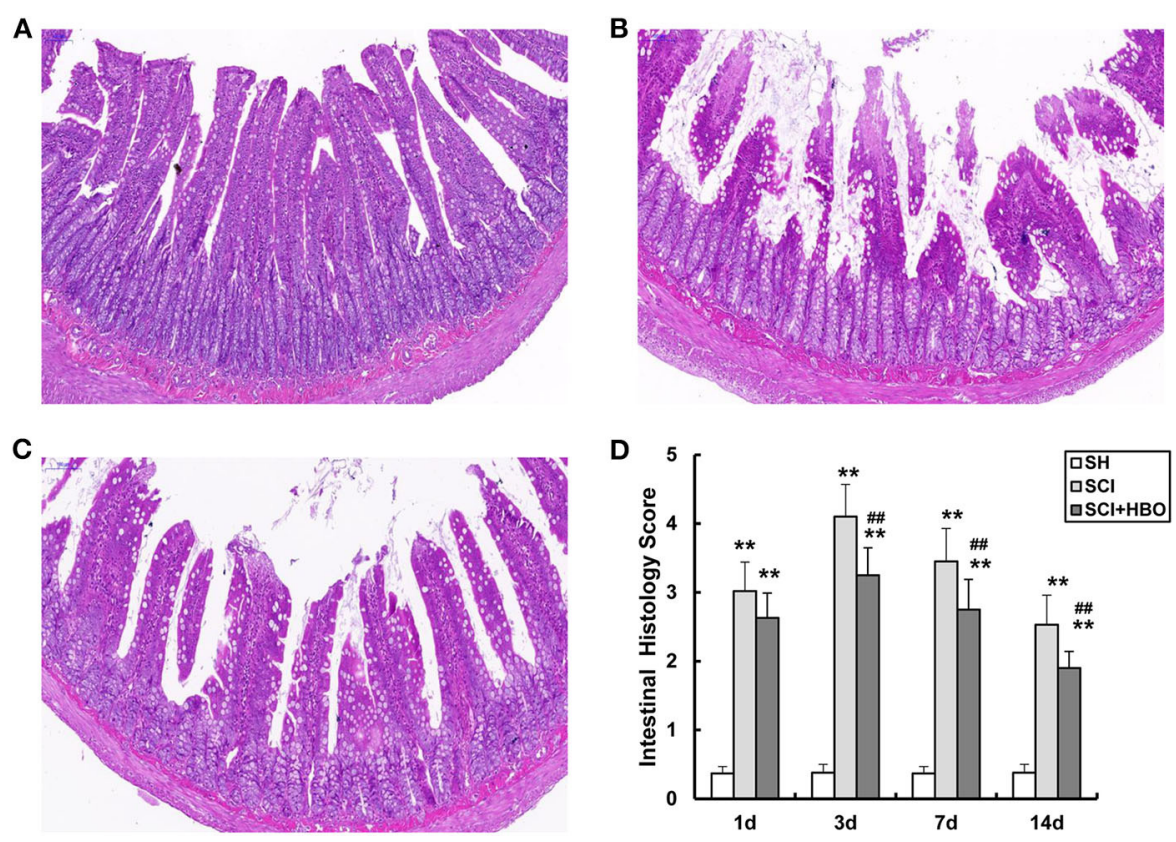

FIGURE 1 | Representative photomicrographs of the jejunum stained with H\&E on postoperative day 3 (all images are $\times 200$ magnification, bar $=100 \mu$ m) and analysis of the jejunum mucosal tissue injury in experiment groups. (A) SH group jejunum showing normal villi and consistent villous height. (B) SCl group jejunum specimens were notable for marked short and disorganized intestinal villi, villi necrosis, and increased crypt depth. (C) SCI+HBO group animals showed no evidence of intestinal villi necrosis nor architectural deformity. (D) The histology score of jejunum. Data are presented as mean \pm SD. Compared with that of the SH group, the histology score of jejunum was significantly higher in the $\mathrm{SCl}$ and $\mathrm{SCl}+\mathrm{HBO}$ groups ( ${ }^{\star \star} P<0.01$ ); HBO treatment significantly decreased the histology score compared with the SCl group (\#\#P<0.01). SH, sham operation; SCl, spinal cord injury; HBO, hyperbaric oxygen.

significantly decreased SCI-induced damage to the jejunal mucosa (Figure 1C). The mucosal injury score of randomly analyzed tissue segments was significantly elevated in the SCI group compared with the SH group $(P<0.01)$, and the rats in the SCI+HBO group exhibited a significantly lower mucosal injury score than that in the SCI group $(P<0.01$, Figure 1D).

To determine whether $\mathrm{HBO}$ treatment has any effects on intestinal barrier permeability after SCI, rats received FITC-labeled dextran $(4 \mathrm{kDa})$ by gavage on day 3 following injury, and the FITC level in blood was measured. Intestinal permeability significantly increased after SCI. Remarkably, HBO treatment protected against injuryinduced intestinal leakage (Figure 2). Taken together, these results demonstrated that $\mathrm{HBO}$ treatment improved intestinal barrier function.

\section{Hyperbaric Oxygen Treatment Increases the Expression of Occludin and ZO-1 After Spinal Cord Injury in Rats}

To assess the influence of $\mathrm{HBO}$ treatment on TJ proteins after SCI, we detected the expression of occludin and ZO1 in jejunum tissue by immunohistochemistry and western blotting. For SCI rats, the expression levels of occludin and $\mathrm{ZO}-1$ was significantly decreased compared with those of the SH group $(P<0.01)$, which decreased to a minimum level on postoperative day 3. However, for rats in the SCI+HBO

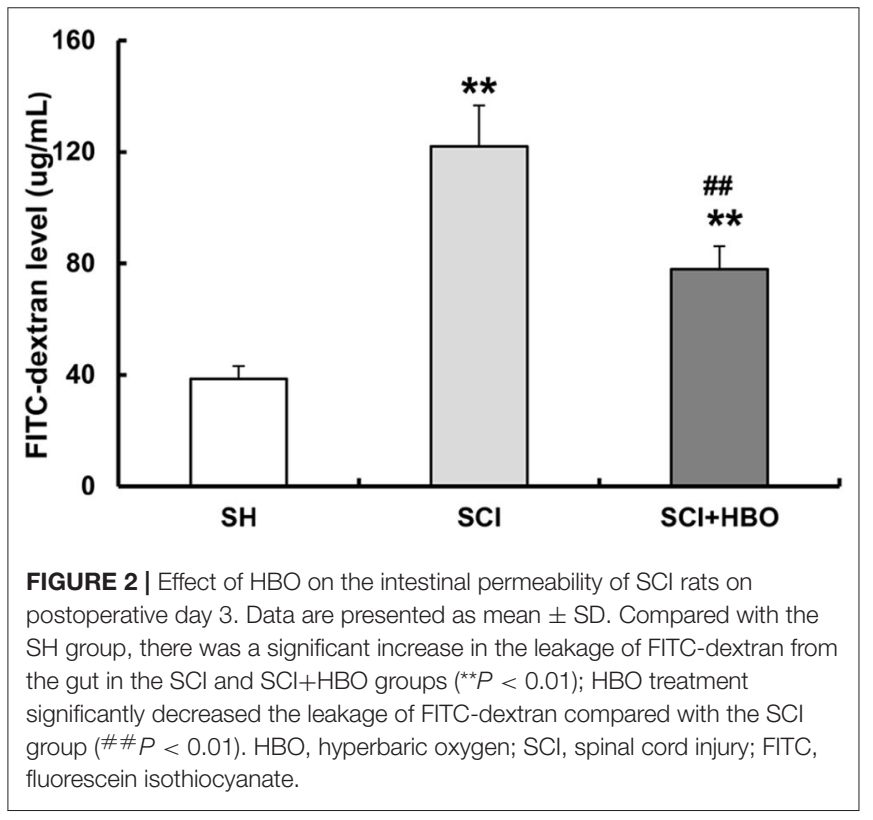

group, the expression of these two proteins was higher than that in the SCI group on days 3, 7, and 14 postoperatively $(P<0.05, P<0.01)$. Figure 3 shows the positive staining of occludin and ZO-1 detected by immunohistochemical method 

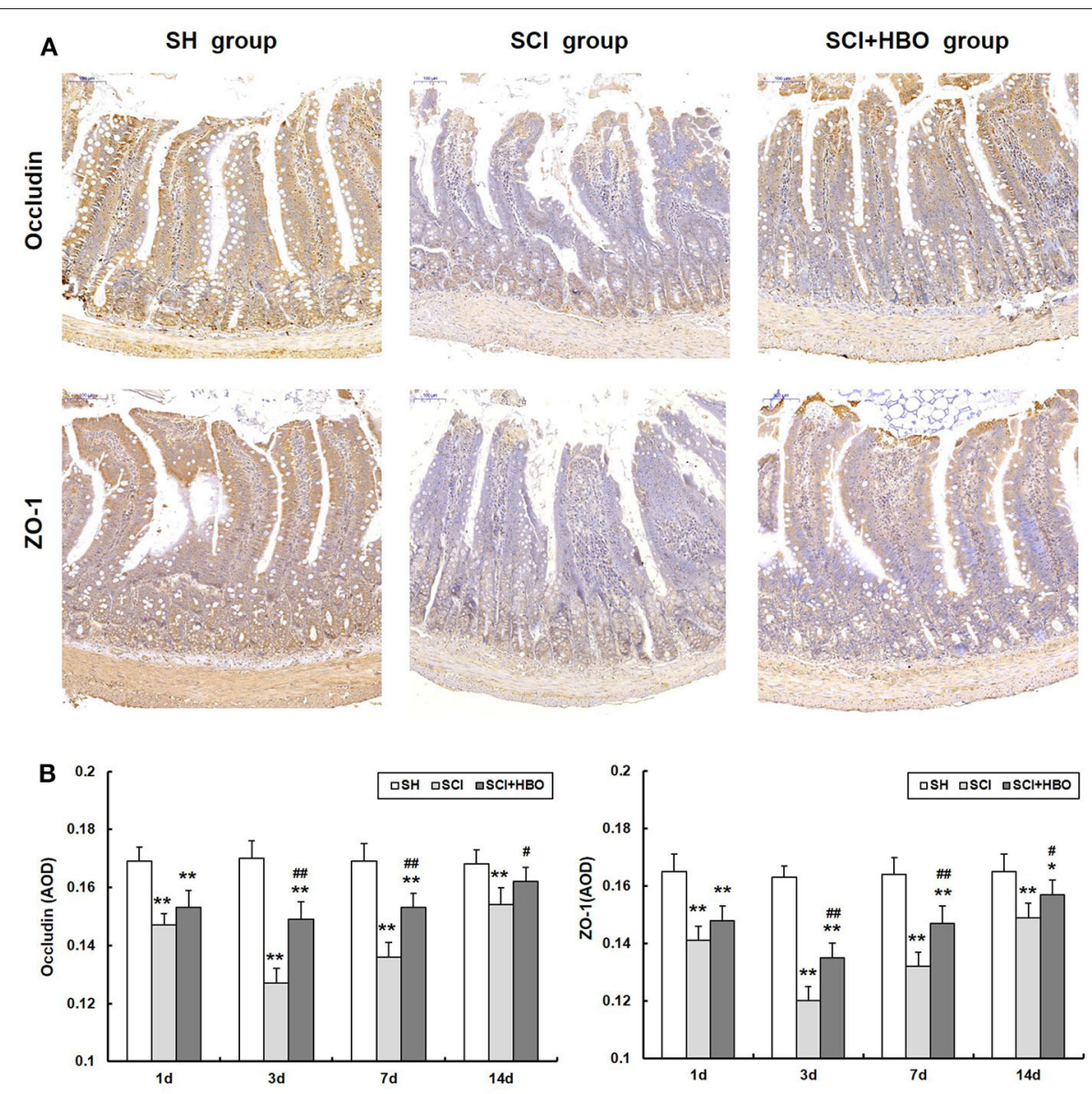

FIGURE 3 | Effect of HBO on occludin and ZO-1 immunoreactivity in jejunum tissue after SCl. (A) Expressions of occludin and ZO-1 in the jejunum tissue visualized by immunohistochemistry under a high magnification on postoperative day 3 (all images are $\times 200$ magnification, bar $=100 \mu \mathrm{m}$ ). (B) Analysis of the average optical density of occludin and ZO-1. Data are presented as mean \pm SD. Compared with the SH group, the average optical density of two factors significantly decreased after $\mathrm{SCl}\left({ }^{\star} P<0.05,{ }^{\star \star} P<0.01\right)$; HBO exposure significantly increased the average optical density of occludin and ZO-1 compared with the SCl group (\# $P<0.05$, $\# \# P<0.01)$. $\mathrm{HBO}$, hyperbaric oxygen; SCl, spinal cord injury.

in different experimental groups, and the analysis of AOD. Figure 4 shows the protein expression level of occludin and ZO-1 determined by western blotting. The results suggested that $\mathrm{HBO}$ treatment upregulated the expression of TJ proteins after SCI.

\section{Hyperbaric Oxygen Treatment Downregulates the Expression of Rho and ROCK1 After Spinal Cord Injury in Rats}

In order to investigate whether HBO treatment influences the Rho/ROCK signal pathway, we analyzed the expression of Rho and ROCK1 in different experimental groups. As shown in Figures 5, 6, in the SCI group, the expression of Rho and ROCK1 was higher than that in the $\mathrm{SH}$ group $(P<0.01)$ and peaked on day 3 postoperatively. In contrast, the expression of Rho and ROCK1 was attenuated on days 3, 7, and 14 in the SCI+HBO group $(P<0.05, P<0.01)$. These results suggested that $\mathrm{HBO}$ treatment inhibited the expression of Rho and ROCK1 after SCI.

\section{Hyperbaric Oxygen Treatment Improves Locomotor Recovery After Spinal Cord Injury in Rats}

Limb locomotor of rats was assessed using $\mathrm{BBB}$ rating scale to examine the effect of $\mathrm{HBO}$ treatment on locomotor recovery after SCI. Compared with the SH group, limb locomotor score of rats significantly decreased at the beginning and gradually increased over time in the SCI and SCI+HBO groups $(P<$ 0.01). Rats treated with $\mathrm{HBO}$ exhibited a more rapid and greater improvement of locomotion than SCI rats. On days 7 and 14 postoperatively, the score in the SCI+HBO group was significantly higher than that in the SCI group $(P<0.01$, Figure 7). These results indicated that $\mathrm{HBO}$ treatment promoted the recovery of motor function after SCI in rats.

\section{Correlations Between Locomotor Recovery and Intestinal Mucosal Injury}

We assessed whether locomotor recovery was linked to intestinal mucosal tissue injury by Pearson's correlation analysis. There 


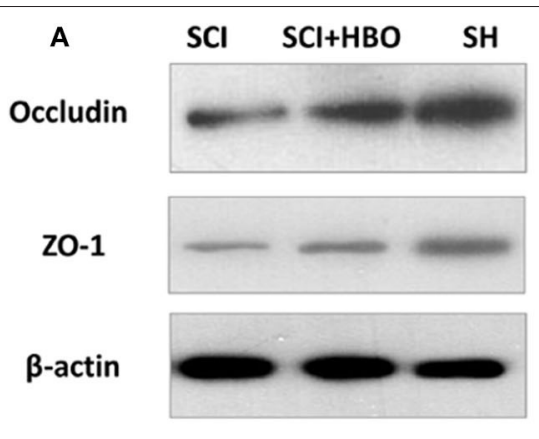

1d

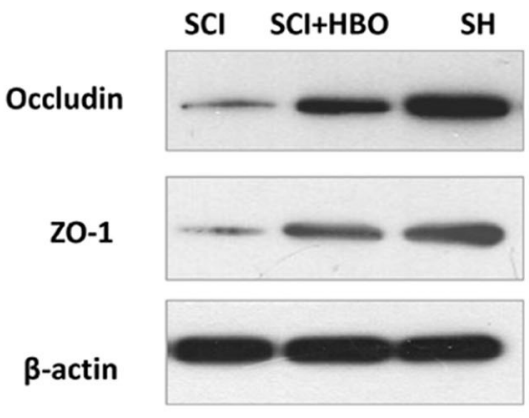

3d
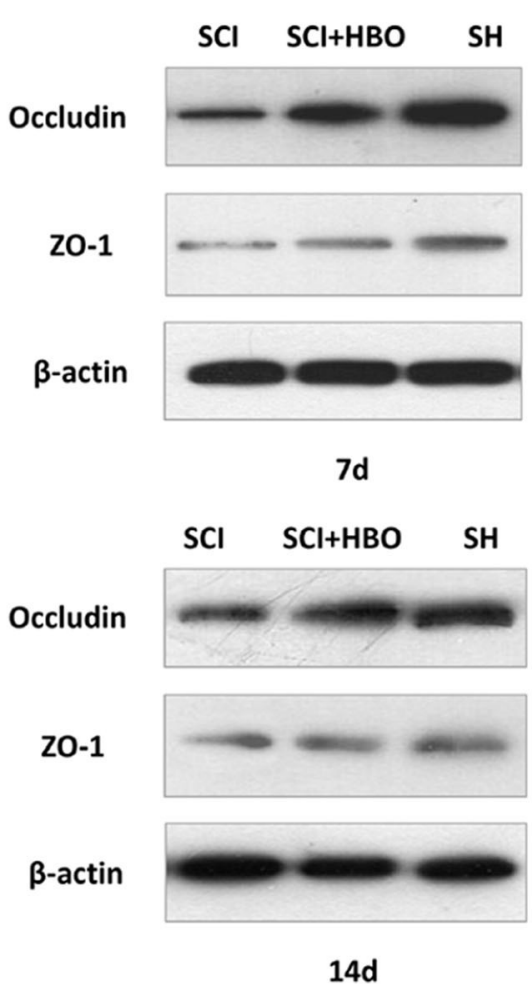
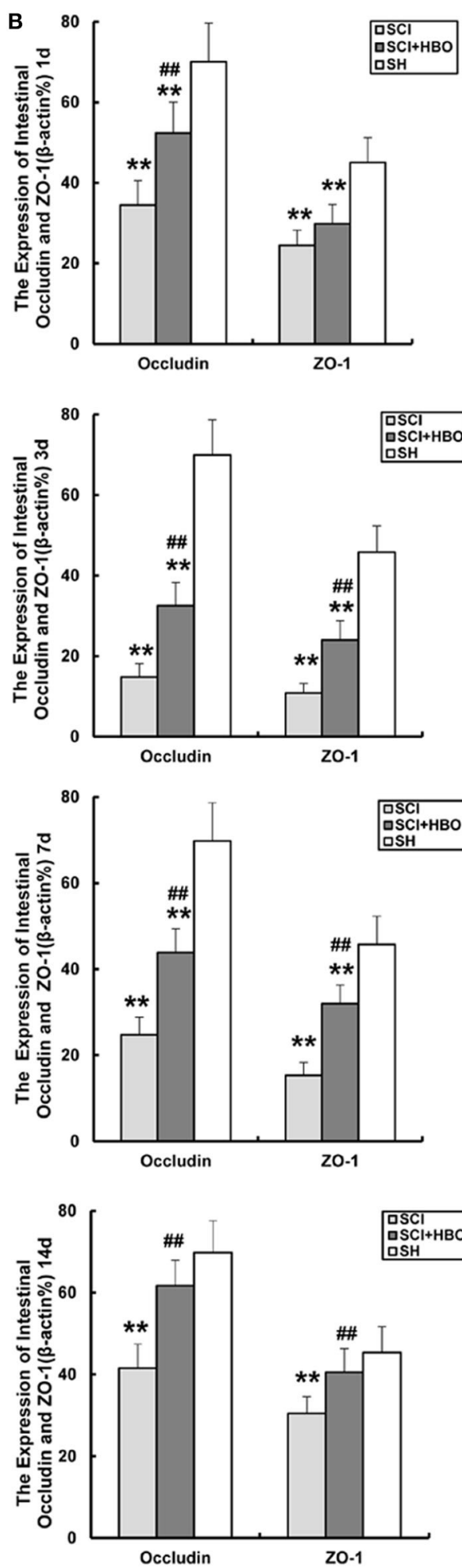

FIGURE 4 | Effect of $\mathrm{HBO}$ on occludin and ZO-1 protein expression in jejunum tissue after SCl. (A) Representative immunoblot of two factors at different time points postoperatively. (B) Analysis of two factors of protein expression. Data are presented as mean $\pm \mathrm{SD}$. Compared with the SH group, the protein expression of two factors significantly decreased in the $\mathrm{SCl}$ and $\mathrm{SCl}+\mathrm{HBO}$ groups $\left({ }^{\star *} \mathrm{P}<0.01\right)$; $\mathrm{HBO}$ treatment significantly increased their protein expressions compared with the $\mathrm{SCl}$ group (\#\# $P<0.01$ ). HBO, hyperbaric oxygen; $\mathrm{SCl}$, spinal cord injury; $\mathrm{SH}$, sham operation. 

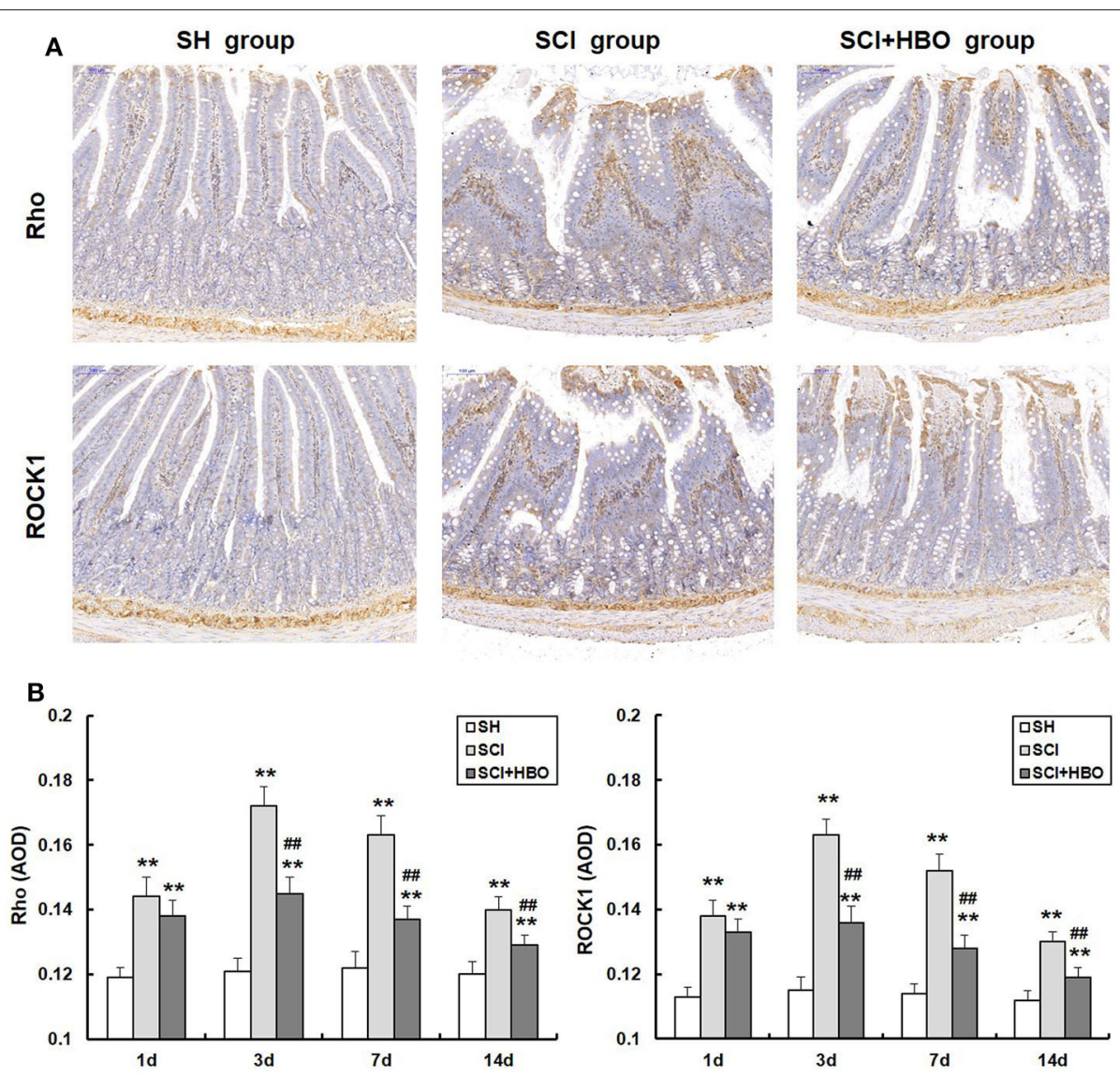

FIGURE 5 | Effect of HBO on Rho and ROCK1 immunoreactivity in jejunum tissue after SCI. (A) Expressions of Rho and ROCK1 in the jejunum tissue visualized by immunohistochemistry under a high magnification on postoperative day 3 (all images are $\times 200$ magnification, bar $=100 \mu \mathrm{m}$ ). (B) Analysis of the average optical density of Rho and ROCK1. Data are presented as mean \pm SD. Compared with the SH group, the average optical density of two factors significantly increased after $\mathrm{SCl}\left({ }^{* *} P<0.01\right.$ ); $\mathrm{HBO}$ exposure significantly decreased the average optical density of Rho and ROCK1 compared with the SCl group (\#\# $P<0.01$ ). HBO, hyperbaric oxygen; SCl, spinal cord injury.

was a significant negative correlation between BBB score and intestinal mucosal injury score in rats in the SCI+HBO group on days 7 and 14 postoperatively $(P<0.05)$; thus, greater locomotor recovery was significantly associated with less damage to intestinal mucosal tissue after $\mathrm{HBO}$ treatment (Figure 8).

\section{DISCUSSION}

Intestinal barrier dysfunction is a common complication of SCI, which severely reduces the quality of life of individuals with SCI $(8,21)$. Although few studies have shown that HBO treatment could protect intestinal barrier function in several animal models (22-24), it has not yet been investigated whether $\mathrm{HBO}$ treatment influences intestinal barrier function of SCI rats. In the present study, we examined the effect of $\mathrm{HBO}$ treatment on intestinal barrier function in a rat model of SCI. HBO treatment alleviated intestinal mucosal injury and protected intestinal barrier function. HBO treatment promoted the expression of TJ proteins to maintain the integrity of the intestinal mucosal barrier possibly by inhibiting the Rho/ROCK signaling pathway.
In addition, we found that locomotor recovery of SCI rats was correlated with the degree of intestinal mucosal injury.

$\mathrm{TJ}$ is an important component and structural basis of intestinal mechanical barrier, which determines the intercellular permeability (25). A number of studies have shown that intestinal mechanical barrier could be strengthened by regulating TJ proteins. Park et al. demonstrated that theaflavins could increase intestinal barrier of Caco-2 Cell monolayers through increasing the expression of AMP-activated protein kinase-mediated occludin and ZO-1 (26). Peng et al. found that qihuang decoction could improve intestinal permeability through promoting the expression of TJ proteins after gastrectomy in rats (27), while following trauma and hemorrhagic shock, the metabolic changes of mucosal layer disrupted TJ protein expression, which could damage intestinal barrier and increase intestinal permeability (13).

The redistribution of systemic blood following SCI resulted in intestinal ischemia and hypoxia, which also disturbed the expression of TJ proteins and increased intestinal permeability, leading to intestinal barrier damage. After that, the bacteria 

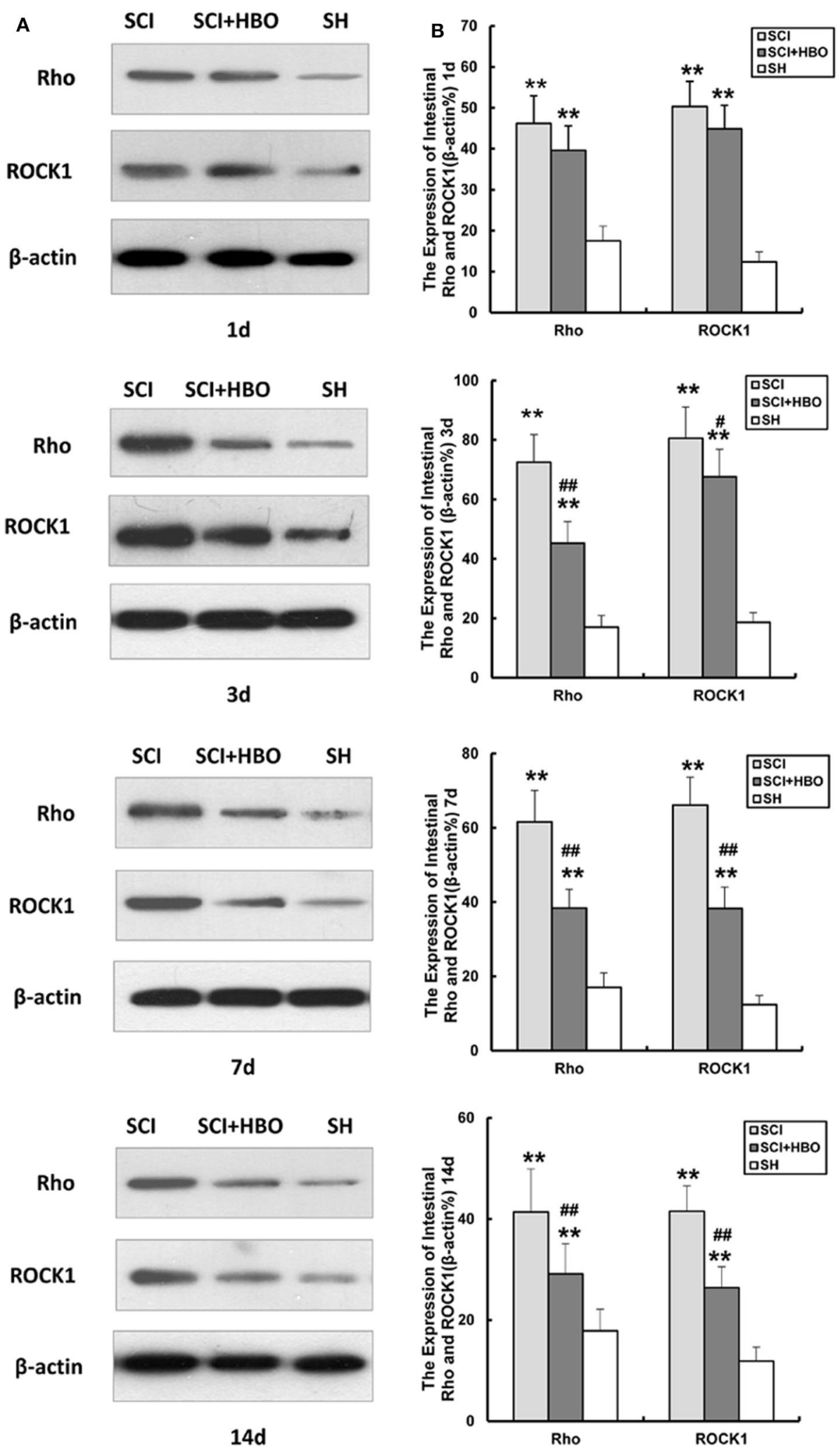

FIGURE 6 | Effect of HBO on Rho and ROCK1 protein expression in jejunum tissue after SCl. (A) Representative immunoblot of two factors at different time points postoperatively. (B) Analysis of two factors of protein expression. Data are presented as mean \pm SD. Compared with the SH group, the protein expression of two factors significantly increased in the $\mathrm{SCl}$ and $\mathrm{SCl}+\mathrm{HBO}$ groups $\left({ }^{\star \star} P<0.01\right)$; $\mathrm{HBO}$ treatment significantly decreased their protein expressions compared with the $\mathrm{SCl}$ group (\#P<0.05, \#\#P<0.01). HBO, hyperbaric oxygen; SCl, spinal cord injury; $\mathrm{SH}$, sham operation. 


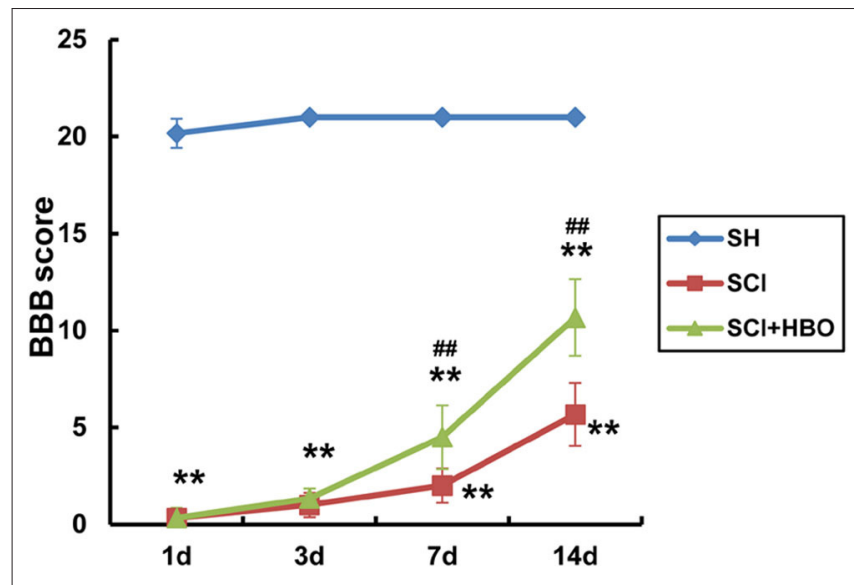

FIGURE 7 | Evaluation of locomotor function of rats using BBB score in experimental groups at destined time points. Compared with that of the $\mathrm{SH}$ group, the BBB score of rats significantly decreased in the $\mathrm{SCl}$ and $\mathrm{SCl}+\mathrm{HBO}$ groups at all destined time points $\left({ }^{\star \star} P<0.01\right)$; HBO treatment significantly increased the BBB score compared with the $\mathrm{SCl}$ group on days 7 and 14 postoperatively (\#\#P<0.01). BBB, Basso-Beattie-Bresnahan; $\mathrm{SH}$, sham operation; $\mathrm{SCl}$, spinal cord injury; $\mathrm{HBO}$, hyperbaric oxygen.

and endotoxins shifted from intestine into blood, which caused enterogenous systemic infection and sepsis, even SIRS and MODS, and seriously influenced the patient's condition $(21,28)$. Consistent with previous studies, in the present study, intestinal mucosal injury score of rats was significantly increased after SCI. In addition, we found that the expression of occludin and ZO-1 was decreased, in parallel with an increase in intestinal permeability, which demonstrated that intestinal barrier was damaged after SCI.

As a noninvasive form of physical therapy, HBO treatment could protect intestinal barrier function though multiple mechanisms in several animal models. In a rat model of ischemia/reperfusion injury, $\mathrm{HBO}$ treatment improved intestinal barrier function by raising the partial pressure of oxygen and oxygen supply in blood, maintaining ATP and aerobic metabolism, and inhibiting TNF- $\alpha$ expression, therefore preventing intestinal epithelium from apoptosis (24). HBO treatment could activate genes encoding TJ proteins to maintain the integrity of intestinal barrier in colitis mice (22). In the sepsis rat model, $\mathrm{HBO}$ treatment alleviated intestinal barrier dysfunction of rats by altering the activation of $N F-\kappa B$, nitric oxide, and myeloperoxidase (23). However, in the SCI rat model, it is unclear whether $\mathrm{HBO}$ treatment influences intestinal barrier function. In the current study, we found that $\mathrm{HBO}$ treatment significantly ameliorated intestinal mucosal injury of SCI rats. Improved intestinal barrier integrity was demonstrated by reduced gut leakage and restored expression of occludin and $\mathrm{ZO}-1$ in $\mathrm{HBO}$-treated rats. The underlying mechanism might involve the improvement of intestinal hypoperfusion and hypoxia after $\mathrm{HBO}$ treatment, which inhibited the decomposition and redistribution of TJ proteins (29). Detailed mechanisms of $\mathrm{HBO}$ treatment regulating the expression of occludin and $\mathrm{ZO}-1$ remain to be clarified.

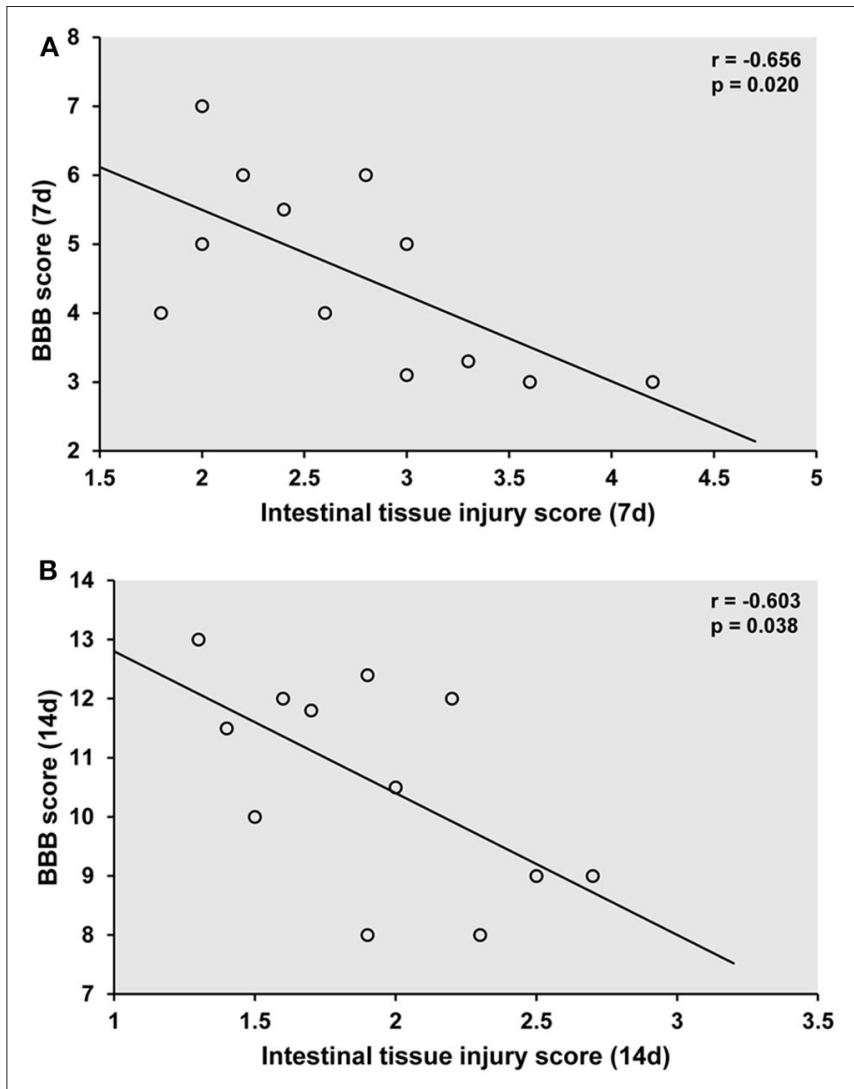

FIGURE 8 | Association of locomotor recovery with intestinal mucosal tissue injury on postoperative days 7 (A) and 14 (B) in the $\mathrm{SCl}+\mathrm{HBO}$ group. Pearson analysis showing the significant negative correlation between the recovery of locomotor function after $\mathrm{HBO}$ treatment and the degree of intestinal mucosal tissue injury in the $\mathrm{SCl}+\mathrm{HBO}$ group $(P<0.05)$. SCl, spinal cord injury; $\mathrm{HBO}$, hyperbaric oxygen.

Precise regulation of $\mathrm{TJ}$ proteins is essential to maintain intestinal barrier function. The Rho/ROCK signaling pathway plays an important role in the regulation of TJ proteins. Peng et al. found that qihuang decoction increased the expression of TJ proteins through inhibiting the Rho/ROCK signaling pathway after gastrectomy (27). In another study, Kim et al. demonstrated that the Rho/ROCK signaling pathway mediated the effect of hyaluronan on the expression of ZO-1 in mice (30). To investigate whether the Rho/ROCK signaling pathway was involved in $\mathrm{HBO}$ modulation on TJ proteins after SCI, we analyzed the expression of Rho and ROCK1 in different experimental groups. Compared with the SH group, SCI induced upregulation of Rho and ROCK1 expression in the intestinal tissue and downregulated the expression of occludin and $\mathrm{ZO}-1$ $(P<0.01)$. Moreover, HBO treatment inhibited the expression of Rho and ROCK1 and increased the expression of occludin and ZO-1 accordingly $(P<0.05, P<0.01)$. These results indicated that $\mathrm{HBO}$ treatment increases the expression of $\mathrm{TJ}$ proteins maybe through inhibiting the Rho/ROCK signaling pathway, which helps to improve intestinal barrier dysfunction induced by SCI in rats (Figure 9). The possible mechanism of 


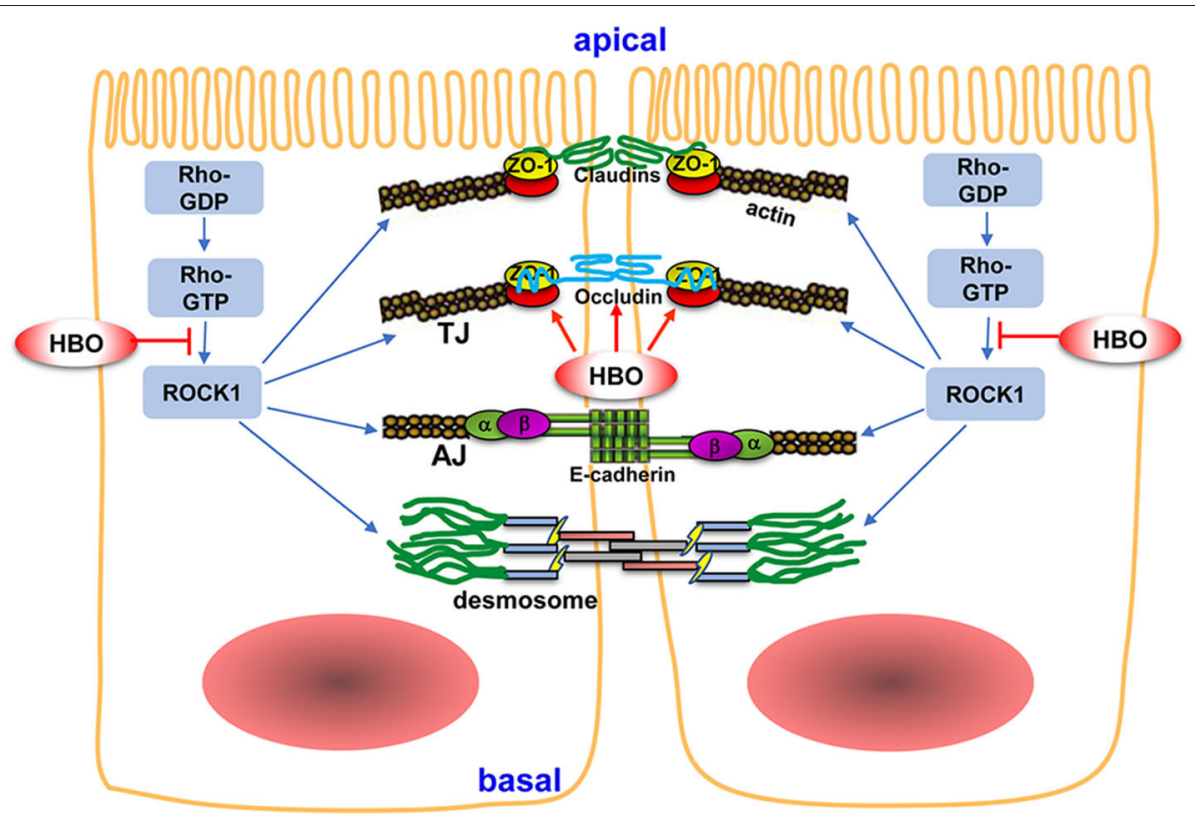

FIGURE 9 | The potential mechanism of HBO treatment on intestinal barrier after SCl. The intestinal mechanical barrier mainly consisted of intact intestinal epithelial cells and their close connections. The connections between adjacent epithelial cells include tight junctions (TJs), adherent junctions (AJs), and desmosomes. TJs are mainly composed of claudins, occludin, and ZO. TJs are regulated by Rho/ROCK signaling pathway. HBO treatment increased the expression of occludin and ZO-1 following SCl and improved intestinal barrier integrity. Furthermore, HBO treatment significantly inhibited Rho/ROCK signaling pathway, which may be the mechanism of $\mathrm{HBO}$ treatment on regulating TJ proteins. Arrows indicate activation and lines with bars inhibition. For details, see text. HBO, hyperbaric oxygen; SCI, spinal cord injury.

HBO induced downregulation of the Rho/ROCK pathway might be that increased oxygen content inhibited the activation of Rho and ROCK in intestinal tissue after HBO treatment. In addition, oxidative stress was probably involved in this process $(31,32)$.

A lot of studies have indicated that translocated bacteria and endotoxins through damaged intestinal barrier induced systematic inflammation, which mediates the secondary injury after SCI and influences the recovery of neurological function (33-35). Meanwhile, these studies also hinted that protecting intestinal barrier might alleviate the degree of secondary injury after SCI. Previous studies have demonstrated that HBO treatment alleviated the inflammation and improved the recovery of neurological function after SCI (36-38). In present study, we also verified that $\mathrm{HBO}$ treatment improved locomotor recovery after SCI in rats. Furthermore, locomotor recovery was negatively correlated with intestinal mucosal injury after HBO treatment. These results further hinted that protecting intestinal barrier function and reducing intestinal mucosal injury maybe help in the recovery of neurological function after SCI.

\section{CONCLUSIONS}

HBO treatment significantly improved neurological function, alleviated intestinal mucosal injury, maintained intestinal barrier integrity, and increased the expression of occludin and ZO1 following SCI. Furthermore, HBO treatment significantly inhibited the Rho/ROCK signaling pathway, which might be the underlying mechanism involved in the regulation of $\mathrm{TJ}$ proteins by $\mathrm{HBO}$ treatment. In addition, locomotor recovery was negatively correlated with intestinal mucosal damage after $\mathrm{HBO}$ treatment. As far as we know, this study, for the first time, explored the effect of $\mathrm{HBO}$ treatment on intestinal barrier following SCI and the underlying mechanisms. These findings not only help to understand the molecular mechanisms of $\mathrm{HBO}$ treatment on improving intestinal barrier function but also provide the theoretical foundation for the clinical application of HBO treatment in GI complications following SCI. However, our study does have limitations. We only demonstrated that $\mathrm{HBO}$ treatment regulates the expression of TJ proteins and the Rho/ROCK signaling pathway after SCI, but the detailed mechanism of $\mathrm{HBO}$ treatment regulating their expression needs to be further investigated. Moreover, further studies are needed to fully translate these findings to human patients in order to expand the clinical application of $\mathrm{HBO}$ in SCI patients.

\section{DATA AVAILABILITY STATEMENT}

The raw data supporting the conclusions of this article will be made available by the authors, without undue reservation. 


\section{ETHICS STATEMENT}

The animal study was reviewed and approved by the Committee for experimental animal welfare and ethics, Capital Medical University.

\section{AUTHOR CONTRIBUTIONS}

XL, JY, and NK designed the experiments. XL, FL, and JZ performed the experiment and acquired the data. XL and

\section{REFERENCES}

1. Goodus MT, Sauerbeck AD, Popovich PG, Bruno RS, McTigue DM. Dietary green tea extract prior to spinal cord injury prevents hepatic iron overload but does not improve chronic hepatic and spinal cord pathology in rats. $J$ Neurotrauma. (2018) 35:2872-82. doi: 10.1089/neu.2018.5771

2. Hou S, Rabchevsky AG. Autonomic consequences of spinal cord injury. Compr Physiol. (2014) 4:1419-53. doi: 10.1002/cphy.c130045

3. Jaglal SB, Munce SE, Guilcher SJ, Couris CM, Fung K, Craven BC, et al. Health system factors associated with rehospitalizations after traumatic spinal cord injury: a population-based study. Spinal Cord. (2009) 47:6049. doi: $10.1038 /$ sc. 2009.9

4. Zhang C, Zhang W, Zhang J, Jing Y, Yang M, Du L. Gut microbiota dysbiosis in male patients with chronic traumatic complete spinal cord injury. J Transl Med. (2018) 16:353. doi: 10.1186/s12967-018-1735-9

5. Deitch EA, Xu D, Kaise VL. Role of the gut in the development of injury- and shock induced SIRS and MODS: the gut-lymph hypothesis, a review. Front Biosci. (2006) 11:520-8. doi: 10.2741/1816

6. Bai CH, Liu H, Li SY, Peng P, Ning LN. The protective effects of sacral nerve electrostimulation on intestinal mucosal mechanical barrier in rats with spinal cord injury. Zhong guo Ying Yong Sheng Li Xue Za Zhi. (2014) 30:471-4.

7. Kigerl KA, Mostacada K, Popovich PG. Gut microbiota are disease-modifying factors after traumatic spinal cord injury. Neurotherapeutics. (2018) 15:60-7. doi: 10.1007/s13311-017-0583-2

8. Jing Y, Yang D, Bai F, Zhang C, Qin C, Li D, et al. Melatonin treatment alleviates spinal cord injury-induced gut dysbiosis in mice. J Neurotrauma. (2019) 36:2646-64. doi: 10.1089/neu.2018.6012

9. Wang F, Liang W, Lei C, Kinden R, Sang H, Xie Y, et al. Combination of $\mathrm{HBO}$ and memantine in focal cerebral ischemia: isthere a synergistic effect? Mol Neurobiol. (2015) 52:1458-66. doi: 10.1007/s12035-014-8949-5

10. Li HZ, Chen JF, Liu M, Shen J. Effect of hyperbaric oxygen on the permeability of the blood-brain barrier in rats with global cerebral ischemia/reperfusion injury. Biomed Pharmacother. (2018) 108:1725-30. doi: 10.1016/j.biopha.2018.10.025

11. Yang J, Wang G, Gao C, Shao G, Kang N. Effects of hyperbaric oxygen on MMP-2 and MMP-9 expression and spinal cord edema after spinal cord injury. Life Sci. (2013) 93:1033-8. doi: 10.1016/j.lfs.2013.10.015

12. Caron TJ, Scott KE, Fox JG, Hagen SJ. Tight junction disruption: helicobacter pylori and dysregulation of the gastric mucosal barrier. World J Gastroenterol. (2015) 21:11411-27. doi: 10.3748/wjg.v21.i40.11411

13. Zhu R, Ma XC. Role of metabolic changes of mucosal layer in the intestinal barrier dysfunction following trauma/hemorrhagic shock. Pathol Res Pract. (2018) 214:1879-84. doi: 10.1016/j.prp.2018.08.023

14. Drolia R, Bhunia AK. Crossing the Intestinal Barrier via Listeria Adhesion Protein and Internalin A. Trends Microbiol. (2019) 27:408-25. doi: 10.1016/j.tim.2018.12.007

15. Amin E, Dubey BN, Zhang SC, Gremer L, Dvorsky R, Moll JM, et al. Rhokinase: regulation, (dys)function, and inhibition. Biol Chem. (2013) 394:1399410. doi: 10.1515/hsz-2013-0181

16. Walsh SV, Hopkins AM, Chen J, Narumiya S, Parkos CA, Nusrat A. Rho kinase regulates tight junction function and is necessary for tight junction assembly in polarized intestinal epithelia. Gastroenterology. (2001) 121:56679. doi: 10.1053/gast.2001.27060
ZL undertook the statistical analysis and interpretation of the data. XL wrote the first draft of the manuscript. JY helped to revise the manuscript. All authors read and have approved the final manuscript.

\section{FUNDING}

This study was supported by Beijing Natural Science Foundation (7202055).

17. Wang Y, Wang X, Yang W, Zhao X, Zhang R. Effect of simvastatin on the intestinal Rho/ROCK signaling pathway in rats with sepsis. J Surg Res. (2018) 232:531-8. doi: 10.1016/j.jss.2018.07.016

18. Basso DM, Beattie MS, Bresnahan JC. Graded histological and locomotor outcomes after spinal cord contusion using the NYU weight-drop device versus transaction. Exp Neurol. (1996) 139:244-56. doi: 10.1006/exnr.1996.0098

19. Douhara A, Moriya K, Yoshiji H, Noguchi R, Namisaki T, Kitade M, et al. Reduction of endotoxin attenuates liver fibrosis through suppression of hepatic stellate cell activation and remission of intestinal permeability in a rat non-alcoholic steatohepatitis model. Mol Med Rep. (2015) 11:1693-700. doi: 10.3892/mmr.2014.2995

20. Chiu CJ, Scott HJ, Gurd FN. Intestinal mucosal lesion in low-flow states. II The protective effect of intraluminal glucose as energy substrate. Arch Surg. (1970) 101:484-8. doi: 10.1001/archsurg.1970.01340280036010

21. Myers SA, Gobejishvili L, Saraswat OS, Wilson CG, Andres KR, Riegler AS, et al. Following spinal cord injury, PDE4B drives an acute, local inflammatory response and a chronic, systemic response exacerbated by gut dysbiosis and endotoxemia. Neurobiol Dis. (2019) 124:353-63. doi: 10.1016/j.nbd.2018.12.008

22. Novak S, Drenjancevic I, Vukovic R, Kellermayer Z, Cosic A, Tolusic LM, et al. Anti-inflammatory effects of hyperbaric oxygenation during DSSinduced colitis in BALB/c mice include changes in gene expression of HIF-1 $\alpha$, proinflammatory cytokines, and antioxidative enzymes. Mediators Inflamm. (2016) 2016:7141430. doi: 10.1155/2016/7141430

23. Sakoda M, Ueno S, Kihara K, Arikawa K, Dogomori H, Nuruki K, et al. A potential role of hyperbaric oxygen exposure through intestinal nuclear factor-kappa B. Crit Care Med. (2004) 32:1722-9. doi: 10.1097/01.ccm.0000132898.27101.6c

24. Zhou SH, Sun YF, Wang G. Effects of hyperbaric oxygen on intestinal mucosa apoptosis caused by ischemia-reperfusion injury in rats. World J Emerg Med. (2012) 3:135-40. doi: 10.5847/wjem.j.1920-8642.2012. 02.010

25. Chen D, Li L, Yan J, Yang X, You Y, Zhou Y, et al. The loss of alpha SNAP downregulates the expression of occludin in the intestinal epithelial cell of acute pancreatitis model. Pancreatology. (2014) 14:347-55. doi: 10.1016/j.pan.2014.06.007

26. Park HY, Kunitake Y, Hirasaki N, Tanaka M, Matsui T. Theaflavins enhance intestinal barrier of Caco-2 Cell monolayers through the expression of AMPactivated protein kinase-mediated Occludin, Claudin-1, and ZO-1. Biosci Biotechnol Biochem. (2015) 79:130-7. doi: 10.1080/09168451.2014.951027

27. Peng H, Shen Y, Zhang Q, Liu J, Wang Z, Huang L, et al. Qihuang decoction promotes the recovery of intestinal immune barrier dysfunction after gastrectomy in rats. Am J Transl Res. (2018) 10:827-36.

28. Besecker EM, Deiter GM, Pironi N, Cooper TK, Holmes GM. Mesenteric vascular dysregulation and intestinal inflammation accompanies experimental spinal cord injury. Am J Physiol Regul Integr Comp Physiol. (2017) 312:R146-56. doi: 10.1152/ajpregu.00347.2016

29. Qi H, Wang P, Liu C, Li M, Wang S, Huang Y, et al. Involvement of HIF-1a in MLCK-dependent endothelial barrier dysfunction in hypoxia. Cell Physiol Biochem. (2011) 27:251-62. doi: 10.1159/000327951

30. Kim Y, West GA, Ray G, Kessler SP, Petrey AC, Fiocchi C, et al. Layilin is critical for mediating hyaluronan $35 \mathrm{kDa}$-induced intestinal epithelial tight 
junction protein ZO-1 in vitro and in vivo. Matrix Biol. (2018) 66:93-109. doi: 10.1016/j.matbio.2017.09.003

31. Yang G, Qian C, Wang N, Lin C, Wang Y, Wang G, et al. Tetramethylpyrazine protects against oxygen-glucose deprivation-induced brain microvascular endothelial cells injury via rho/rho-kinase signaling pathway. Cell Mol Neurobiol. (2017) 37:619-33. doi: 10.1007/s10571-0160398-4

32. Singh J, Kumar S, Krishna CV, Rattan S. Aging-associated oxidative stress leads to decrease in IAS tone via RhoA/ROCK downregulation. Am J Physiol Gastrointest Liver Physiol. (2014) 306:G983-991. doi: 10.1152/ajpgi.00087.2014

33. Mukhamedshina YO, Akhmetzyanova ER, Martynova EV, Khaiboullina SF, Galieva LR, Rizvanov AA. Systemic and local cytokine profile following spinal cord injury in rats: a multiplex analysis. Front Neurol. (2017) 8:581. doi: 10.3389/fneur.2017.00581

34. Stammers AT, Liu J, Kwon BK. Expression of inflammatory cytokines following acute spinal cord injury in a rodent model. J Neurosci Res. (2012) 90:782-90. doi: 10.1002/jnr. 22820

35. Kigerl KA, Hall JC, Wang L, Mo X, Yu Z, Popovich PG. Gut dysbiosis impairs recovery after spinal cord injury. J Exp Med. (2016) 213:260320. doi: $10.1084 /$ jem. 20151345
36. Geng CK, Cao HH, Ying X, Zhang HT, Yu HL. The effects of hyperbaric oxygen on macrophage polarization after rat spinal cord injury. Brain Res. (2015) 1606:68-76. doi: 10.1016/j.brainres.2015.01.029

37. Liang F, Li C, Gao C, Li Z, Yang J, Liu X, et al. Effects of hyperbaric oxygen therapy on NALP3 inflammasome expression in rats after spinal cord injury. Mol Med Rep. (2015) 11:4650-6. doi: 10.3892/mmr.2015.3314

38. Sun L, Zhao L, Li P, Liu X, Liang F, Jiang Y, et al. Effect of hyperbaric oxygen therapy on HMGB1/NF- $\kappa \mathrm{B}$ expression and prognosis of acute spinal cord injury: a randomized clinical trial. Neurosci Lett. (2019) 692:47-52. doi: 10.1016/j.neulet.2018.10.059

Conflict of Interest: The authors declare that the research was conducted in the absence of any commercial or financial relationships that could be construed as a potential conflict of interest.

Copyright (c) 2020 Liu, Liang, Zhang, Li, Yang and Kang. This is an open-access article distributed under the terms of the Creative Commons Attribution License (CC $B Y)$. The use, distribution or reproduction in other forums is permitted, provided the original author(s) and the copyright owner(s) are credited and that the original publication in this journal is cited, in accordance with accepted academic practice. No use, distribution or reproduction is permitted which does not comply with these terms. 